\title{
Die Planung von Lehr-Lern-Einheiten mit digitalen Medien: Konzepte und Befunde
}

\author{
Josef Schrader $\cdot$ Sabine Schöb
}

Eingegangen: 30. August 2016 / Angenommen: 1. Dezember 2016 / Online publiziert: 19. Dezember 2016

(C) Der/die Autor(en) 2016. Dieser Artikel ist eine Open-Access-Publikation.

Zusammenfassung Die Planung von Lehr-Lern-Einheiten gehört zu den Kernaufgaben von Lehrenden in der Erwachsenen- und Weiterbildung. Sie stellt eine regelmäßig wiederkehrende Anforderung dar, die es erfordert, fallbezogene Entscheidungen über die Ziele, die Inhalte, die Medien und die Sozial- und Arbeitsformen von Lehr-Lern-Prozessen mit Blick auf die avisierten Teilnehmenden sowie die organisatorischen und räumlichen Rahmenbedingungen zu treffen. Die empirische Forschung zeigt allerdings, dass u. a. die Interdependenz didaktischer Entscheidungen oft nur unzureichend berücksichtigt wird. Um die Komplexität didaktischer Planungs- und Entscheidungsprozesse zu reduzieren, lassen sich digitale Medien nutzen. Hier setzt die in diesem Beitrag vorgestellte Kursplanungs-App an. Erste empirische Befunde zeigen, dass dieses Tool didaktisch begründete Entscheidungen von Lehrenden unterstützen und damit zu einer größeren Professionalität des Lehrhandelns beitragen kann.

Schlüsselwörter Didaktische Planung · Lehrhandeln · Cognitive Load · Digitale Medien · App

Prof. Dr. J. Schrader $(\bowtie)$

Deutsches Institut für Erwachsenenbildung, Leibniz-Zentrum für Lebenslanges Lernen e.V.,

Heinemannstraße 12-14, 53175 Bonn, Deutschland

E-Mail: schrader@die-bonn.de

S. Schöb $(\bowtie)$

Wirtschafts- und Sozialwissenschaftliche Fakultät, Institut für Erziehungswissenschaft, Abteilung Erwachsenenbildung/Weiterbildung, Eberhard Karls Universität Tübingen,

Münzgasse 11, 72070 Tübingen, Deutschland

E-Mail: sabine.schoeb@uni-tuebingen.de 


\section{The planning of teaching and learning with digital media: concepts and findings}

Abstract The planning of teaching and learning is a key task for teachers of adult and continuing education. This recurrent challenge demands situational decisions regarding objectives, contents, media and activities of teaching and learning, taking into consideration both the learner as well as organisational and spatial factors. However, empirical research indicates that these complex and interdependent decisions are often tackled insufficiently. Digital planning tools can be used to reduce the complexity of didactical decision making. This article presents an app that has been developed to support adult education teachers. Empirical findings point out that this tool may support didactically reasonable decisions and, as a result, may foster the quality of teaching activities.

Keywords Educational planning $\cdot$ Teaching activities $\cdot$ Cognitive load $\cdot$ Digital media $\cdot$ App

\section{Einleitung}

Die Planung von Lehr-Lern-Einheiten gehört neben ihrer Durchführung und Evaluation zu den Kernaufgaben von Lehrkräften aller Bildungsbereiche. Sie erfordert Entscheidungen über die zeitliche, sachliche und soziale Strukturierung (vgl. Bromme 1997), die vorbereitend, aber auch begleitend zu Veranstaltungen getroffen werden müssen. Die didaktische Literatur lenkt die Aufmerksamkeit pädagogischer Praktiker in der Regel auf eine Reflexion von Zielen und Inhalten einzelner Lehr-LernEinheiten sowie auf die dazu ,passenden“ Arbeits- und Sozialformen, medialen Repräsentationen und Formen der (Selbst-)Überprüfung von Lernfortschritten (vgl. Siebert 2006; Schlutz 2006; Sandfuchs 2009). Didaktisch-methodische Entscheidungen stellen insofern komplexe Aufgabenstellungen dar, als die Dimensionen von Lehr-Lern-Prozessen in einem Interdependenzverhältnis stehen (vgl. Heimann et al. 1965), so dass Entscheidungen über Teil-Dimensionen nicht unabhängig voneinander getroffen werden können. Für Lehrkräfte in der Erwachsenen- und Weiterbildung gilt, dass sie solch komplexe Reflexionen auch, oder besser gesagt: gerade dann anstellen müssen, wenn sie didaktische Entscheidungen im Sinne einer Teilnehmerorientierung gemeinsam mit den Lernenden treffen möchten (vgl. Breloer et al. 1980). Diese Anforderung wird dadurch noch gesteigert, dass Lehrkräfte die Teilnehmenden in der Regel erst mit dem Kursbeginn kennenlernen. Auch der Umstand, dass die Erwachsenen- und Weiterbildung sich im Unterschied zu Schule, Berufsbildung und Hochschule in geringerem Maße auf strukturierende Curricula und darauf bezogene Lehrmaterialien stützen kann, verweist neben grundlegenden Gemeinsamkeiten auf einen spezifischen Bedarf an didaktischer Kompetenz. Wie vorliegende Studien zur sozialen und beruflichen Lage von Lehrkräften der Erwachsenen- und Weiterbildung jedoch zeigen, handelt es sich zu einem großen Teil um Quereinsteiger, die ihre Bildungsangebote überwiegend ohne Rekurs auf pädagogisch-psychologisches bzw. erwachsendidaktisches Wissen planen, durchführen und 
evaluieren. Ihre Expertise beruht vor allem auf fachlichem Wissen und beruflicher Erfahrung (vgl. WSF 2005; Martin und Langemeyer 2014; DIE et al. 2016).

Konsequenterweise formulieren Lehrkräfte in Befragungen vor allem einen Bedarf an handlungsleitendem Wissen, das sie bei der zielgruppengerechten und situationsadäquaten Gestaltung von Bildungsangeboten unterstützt (vgl. Schöb et al. 2015). Methodische Ratgeberliteratur und in hohem Maße auch das Internet sind die bevorzugten Informationsquellen bei der Vorbereitung von Veranstaltungen (vgl. Michel 2008). Bei diesen Materialien handelt es sich zumeist um erfahrungsgestütztes „Rezeptwissen“, das von begrenzten Zeitressourcen der Lehrkräfte ausgeht, ihren eher fachlichen als pädagogischen Hintergrund in Rechnung stellt und ,schnelle Lösungen“" verspricht (vgl. Kade 1990). Ein Mangel besteht demgegenüber an erziehungswissenschaftlich fundierten und gleichzeitig praxisnah ausgerichteten Handlungshilfen, d.h. an „Werkzeugen“, die an den Stand der didaktischen Diskussion sowie der Forschung zur Professionalität des Lehrhandelns anschließen und deren Wirksamkeit empirisch geprüft ist.

An diese Ausgangslage knüpft die hier vorgestellte Studie an. Der Beitrag geht von der Annahme aus, dass wissenschaftlich fundierte Planungshilfen didaktisch begründete Entscheidungen von Lehrkräften in der Vorbereitung und bei der Durchführung von Bildungsangeboten dabei unterstützen können, die Interdependenz und Komplexität solcher Entscheidungen angemessen zu berücksichtigen und damit zu einer Steigerung der Professionalität des Lehrhandelns beizutragen. Vorgestellt wird die Entwicklung einer digitalen Kursplanungs-App, die an weithin geteilte Annahmen der didaktischen Diskussion anschließt, zeitlich und örtlich flexibel einsetzbar ist und eine fallbasierte und somit an praktischen Anwendungsszenarien ausgerichtete Planung von Bildungsangeboten erlaubt. Die Frage, inwiefern eine solche Anwendersoftware die Planung von Lehr-Lern-Einheiten erleichtern und gleichzeitig die Qualität der didaktischen Entscheidungen fördern kann, ist Gegenstand einer begleitenden empirischen Feldstudie.

Im Folgenden werden die konzeptionellen Grundlagen und die Nutzungsmöglichkeiten der Kursplanungs-App vorgestellt sowie Befunde aus einer Studie präsentiert, in der ein Prototyp mit angehenden Lehrkräften der Erwachsenen- und Weiterbildung erprobt wurde. Abschließend wird ein Fazit im Blick auf weiterführende Forschungsfragen und Entwicklungsarbeiten sowie eine mögliche Verbreitung der App in die (erwachsenen-)pädagogische Praxis gezogen.

\section{Didaktische Konzepte und empirische Befunde zum Planungshandeln von Lehrkräften}

Die Planung von Lehr-Lern-Einheiten umfasst alle vorbereitenden und begleitenden Überlegungen und Entscheidungen zur Konzeption und Umsetzung einer Veranstaltung mit dem Ziel einer „Orchestrierung“ des Lehrens und Lernens (vgl. Oser und Baeriswyl 2001; Sandfuchs 2009). Sie dient als eine Art „Drehbuch“ für die Durchführung von Veranstaltungen und stellt den Versuch dar, vorab formulierte oder prozessbegleitend gemeinsam mit den Teilnehmenden entwickelte Lehr- und Lernziele in einer spezifischen Form zu erreichen. 
Die Lehr-Lern-Planung gehört zum Kernbestand der didaktischen Literatur. Dabei ist allen didaktischen Modellen gemeinsam, dass sie folgende Grundfragen adressieren: In welcher Absicht tue ich etwas? (Ziele); Was bringe ich in den Horizont der Lernenden? (Inhalte); Wie tue ich das? (Methode); Mit welchen Mitteln verwirkliche ich das? (Medien); An wen vermittle ich das? (Teilnehmende); In welcher Situation vermittle ich das? (organisationaler Rahmen) (grundlegend Heimann 1962). Diese didaktischen Theorien und Modelle gehen dabei meist von einer Interdependenz der angesprochenen Dimensionen aus, die folglich bei der Planung berücksichtigt werden sollte. Dies gilt für die Praxis der Erwachsenen- und Weiterbildung in besonderem Maße, in der Einzelveranstaltungen einen großen Teil des Programmangebots ausmachen, die unter wechselnden organisationalen Bedingungen und für je besondere Adressatengruppen mit oft heterogenen Voraussetzungen und Interessen durchgeführt werden. Planung lässt sich als ein didaktisches Probehandeln begreifen, ein Antizipieren und Durchspielen eines möglichen Kursgeschehens, um auf der Basis durchdachter Vorgehensweisen gerüstet für die Durchführung im „Ernstfall““ zu sein (vgl. Müller et al. 2012).

Während die erziehungswissenschaftliche und erwachsenenpädagogische Literatur ein großes Angebot an analytischen und präskriptiven didaktischen Theorien, Modellen und Prinzipien entwickelt hat, ist das empirische Wissen darüber, wie didaktisches Planungshandeln konkret realisiert wird, durchaus begrenzt (vgl. Stanik 2016 in diesem Heft). Die empirischen Studien, überwiegend aus dem Schulbereich, zeigen u. a., dass vor allem Lehranfänger die Lehr-Lern-Planung als eine anspruchsvolle Aufgabe betrachten, die sie unter hohen Rechtfertigungsbedarf gegenüber Vorgesetzten, Kollegen und Kolleginnen wie auch gegenüber sich selbst stellt (vgl. Wiater 1997; Sandfuchs 2009). Wie die Expertiseforschung in der Lehrerbildung belegt, fällt es Lehrkräften besonders schwer, die Komplexität der Gestaltung von Lehr-Lern-Einheiten zu überblicken sowie ihr eigenes Handeln zu reflektieren. Dies schränkt die Qualität von Lehr-Lern-Prozessen vermutlich schon deshalb ein, weil die immer notwendige situative Flexibilität nur auf der Grundlage einer fundierten Planung gesichert werden kann. Erfahrene Lehrkräfte verfügen hingegen in der Regel über Planungsstrukturen und Handlungsroutinen, die sie weitgehend automatisiert einsetzen, so dass sie sich häufig auf eine verkürzte Planung ihrer Bildungsangebote beschränken und gleichwohl situativ flexibel reagieren können (vgl. Sandfuchs 1982, 2009; Bromme 1997; Gruber 2004).

Blickt man auf den Forschungsstand zur Erwachsenen- und Weiterbildung, so verlassen sich Lehrkräfte bei ihren didaktischen Entscheidungen oft auf individuelle, berufsbiographisch geprägte Erfahrungen und Selbstverständnisse (vgl. Kade 1989; Hof 2001), Alltagsroutinen oder die „Weisheit der Praxis“, wie sie u.a. in einer großen Fülle von Ratgeberliteratur mit teils explizit antiszientistischer Grundhaltung angeboten wird (vgl. Schrader 2010; Hoffmann 2011). So finden sich in einschlägigen Werken der Erwachsenen- und Weiterbildung ausdrücklich formulierte Hinweise, dass didaktische Modelle eine „Idealkonstruktion“ von Unterricht formulieren, die nicht eins zu eins in die Praxis übertragen werden kann, und dass das methodische Handeln daher besser am konkreten Fall ausgerichtet und situativ entschieden werden sollte (vgl. Siebert 2006). Die Ratgeberliteratur kommt den Arbeitsweisen vieler Lehrkräfte insofern entgegen, als sie Methoden- gegenüber 
Inhaltsfragen favorisiert (vgl. Haberzeth 2010). Diese Befunde lassen auf einen Bedarf an Arbeitshilfen schließen, die die Aufgabe des Planens in ihrer Komplexität berücksichtigen und gleichzeitig flexibilisieren können, ohne dass sie als „,̈berforderung“ bzw. als „zeitaufwändiges Übel“ wahrgenommen werden (vgl. Wiater 2009). Aufgrund der Komplexität didaktischen Planens und Entscheidens, die nach den derzeitigen empirischen Befunden immer wieder auch zu ,abkürzenden“ Verfahren führt, lohnt es, über Planungshilfen nachzudenken, die ein Durchspielen, Ausprobieren und Revidieren von Möglichkeiten erlauben und auf der Grundlage didaktischen Wissens begründete Entscheidungen unterstützen (vgl. Müller et al. 2012).

\section{Planungshandeln als eine komplexe Aufgabe - Der Anregungsgehalt der Kognitionspsychologie}

Wenn man, wie oben aufgezeigt, davon ausgeht, dass es - je nach Grad der Expertise der Lehrkräfte - eine mehr oder weniger große Diskrepanz zwischen den Idealvorstellungen didaktischer Modelle und Theorien und dem alltäglich gezeigten Planungshandeln gibt, so ist nach den Ursachen dafür zu fragen. Häufig wird dazu, wie erläutert, auf den geringen Grad der Professionalisierung (erwachsenen-) pädagogischer Handlungsfelder verwiesen. Solche Hinweise sind sicherlich nachvollziehbar. Dabei werden aber möglicherweise Herausforderungen übersehen, die sich aus der Komplexität des Planungshandelns selbst ergeben, insbesondere aus der Interdependenz didaktischer Entscheidungen. Möglicherweise stellen die für die Planung von Lehr-Lern-Einheiten derzeit verfügbaren didaktischen Konzepte keine nützlichen Heuristiken dar, um die kognitiven Prozesse angemessen zu unterstützen, die für eine didaktisch begründete Planung notwendig sind.

Nimmt man die Cognitive Load Theory im Sinne Swellers (1994) zum Ausgangspunkt, so könnten die Gründe dafür in einer Überforderung des Arbeitsgedächtnisses der Planenden liegen. Diese Theorie wurde bisher allerdings vor allem für die Analyse von Lernprozessen genutzt; zudem fehlt es noch an empirischen Befunden zur Unterscheidbarkeit der voneinander unterschiedenen Formen kognitiver Belastung (vgl. Kirschner 2002; Schnotz und Kürschner 2007; Gerjets et al. 2009). Gleichwohl nutzen wir diese Theorie im Folgenden als eine Heuristik, um mögliche kognitive Herausforderungen bei der Planung von Lehr-Lern-Einheiten zu identifizieren und zugleich zu fragen, wie sie durch digitale Medien reduziert werden können. Sweller unterscheidet drei mögliche Formen kognitiver Überlastung.

1. Sweller unterstellt intrinsic cognitive load u. a. dann, wenn das Lernmaterial komplex ist und seine einzelnen Elemente eine hohe Interaktivität aufweisen. Übertragen auf das Planungshandeln könnte sich eine (zu) hohe kognitive Belastung z. B. aus der didaktisch begründeten Anforderung ergeben, Planungsentscheidungen mit Rücksicht auf das Interdependenzverhältnis von Zielen, Inhalten, Methoden, Medien und Lernerfolgskontrollen des Unterrichts zu treffen.

2. Laut Sweller ergibt sich extraneous cognitive load u. a. dann, wenn das Lernmaterial zu komplex dargestellt wird. Für das Planungshandeln könnte sich ein 
hoher extraneous cognitive load dadurch ergeben, dass z. B. die Darstellung von Unterrichtsmethoden zumeist auf schriftlichen Berichten von nur geringer Anschaulichkeit beruht. Eine hohe kognitive Belastung könnte sich zudem aus der Anforderung ergeben, nicht nur ,anschauliche“ und handhabbare, sondern auch evident „wirksame“ Methoden einzusetzen, Unterrichtsmethoden also zugleich sowohl im Blick auf ihre Handhabbarkeit als auch im Blick auf ihre Wirksamkeit zu beurteilen.

3. Schließlich unterstellt Sweller germane cognitive load u. a. dann, wenn die Anforderungen, das Lernmaterial zu verstehen, im Prozess des Lernens zu hoch sind. Übertragen auf das Planungshandeln könnte sich eine (zu) hohe kognitive Belastung z. B. aus der Anforderung ergeben, Unterrichtsentwürfe linear zu strukturieren (Einstieg-Erarbeitung-Sicherung), während der Planungsprozess aber nur iterativ und in einer Mischung aus top-down- und bottom-up-Prozessen bewältigt werden kann (vgl. Sweller 1994; Sweller et al. 1998).

Digitale Medien vermögen alle drei Formen solcher kognitiven Belastungen in Planungsprozessen zu reduzieren. So können mit Hilfe digitaler Medien erstens die Interdependenzbeziehungen zwischen einer Vielzahl didaktischer Dimensionen mit beliebiger Komplexität aufgezeigt und für Auswahlentscheidungen aufbereitet werden. Solche Leistungen überfordern die Kapazität des menschlichen Arbeitsgedächtnisses insbesondere dann, wenn mehrere Dimensionen mit jeweils mehreren Unterkategorien gleichzeitig berücksichtigt werden sollen (z. B. die „Passung“ von Methoden zu Gruppengröße, Zeitfenstern und Lernzielen). Zudem können, zweitens, multimodal gestaltete Informationen beispielsweise zu Unterrichtsmethoden in einer Kombination von Bild- und Ton- mit Textmaterialien zur Anschaulichkeit beitragen, indem sie mehrere Sinne und Informationskanäle der menschlichen Wahrnehmung ansprechen (z. B. mit Hilfe authentischer Unterrichtsvideos). Darüber hinaus kann, drittens, eine nicht-lineare Präsentation digitaler Lernmaterialien das Arbeitsgedächtnis dadurch entlasten, dass didaktische Dimensionen und Unterrichtsentwürfe iterativ und spielerisch erschlossen werden; dies könnte die Flexibilität des Planungshandelns und damit auch die Lernmotivation erhöhen (vgl. Mayer und Anderson 1992; Moreno und Valdez 2005). Insgesamt bieten digitale Medien in dieser Lesart die Möglichkeit, kognitive Kapazitäten für didaktische Reflexionen im Planungsprozess ,frei“ zu halten, wodurch die Umsetzung von Planungsentwürfen und der Transfer des Gelernten in die Praxis positiv beeinflusst werden könnten (vgl. Lave und Wenger 1991).

\section{Die Entwicklung einer App zur videogestützten Kursplanung}

In einem zugleich grundlagenwissenschaftlich fundierten und auf Anwendung zielenden Vorgehen wurde die Entwicklung der Kursplanungs-App zunächst auf einen Prototyp zur Gestaltung von Einstiegssituationen beschränkt, die gemäß der Befragung von Schöb et al. (2015) für Lehrkräfte der Erwachsenen- und Weiterbildung eine wiederkehrende und zentrale Anforderung darstellen. Ausgehend von diesem Bedarf wurden auf der Basis einer umfassenden Literaturrecherche 18 für die Ge- 
Abb. 1 Vorselektierte Ansicht der Kursplanungs-App in Form einer tag cloud. (Quelle: Eigene Darstellung)

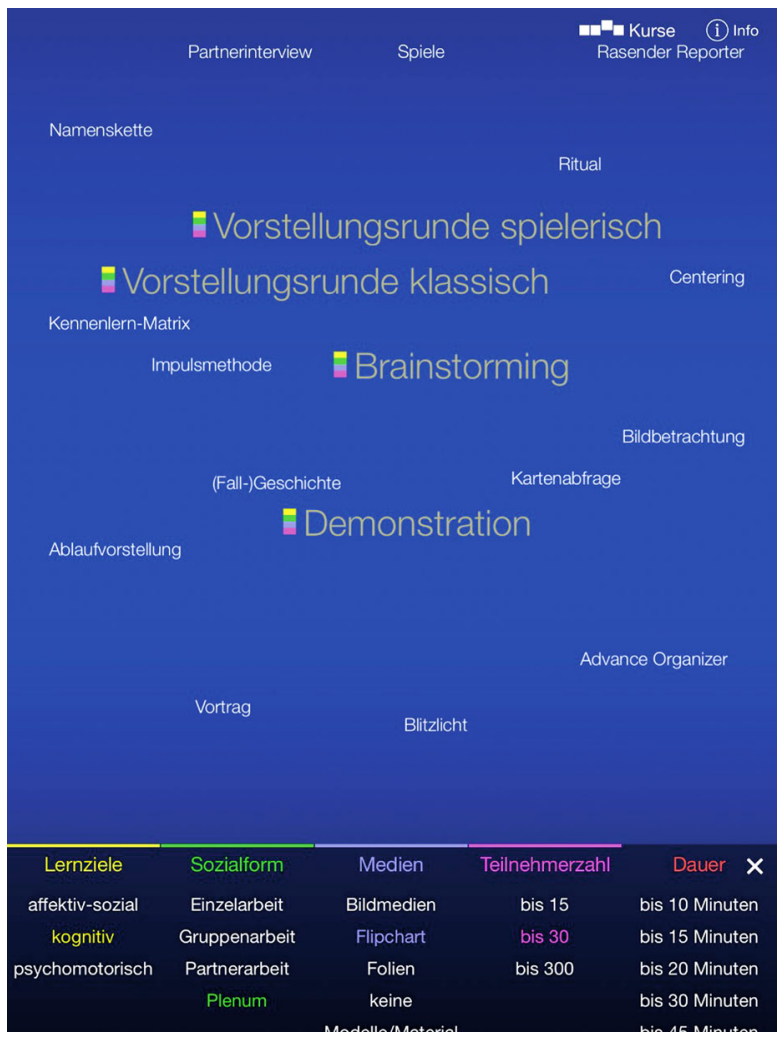

staltung von Einstiegssituationen typische Methoden zusammengestellt und im Blick auf Aktualität und Passung von zwei Experten aus der Allgemeinen Didaktik bzw. der Erwachsenenbildung bewertet. Dieses Set an Methoden wurde auf der Grundlage der im vorhergehenden Kapitel knapp skizzierten kognitionspsychologischen Überlegungen und Befunde für die Entwicklung einer Kursplanungs-App für Lehrende genutzt. Ausgewählte, aber zentrale Funktionalitäten dieser App werden im Folgenden beschrieben.

Um den intrinsic cognitive load bei Planungsprozessen zu reduzieren, bietet die App die Möglichkeit, Interdependenzbeziehungen zwischen didaktischen Dimensionen schnell sichtbar zu machen (vgl. Mayer und Moreno 2010; Abb. 1). Die prinzipiell verfügbaren Methoden können anhand von Vorentscheidungen der Lehrkräfte über die angestrebten Lernziele, die Sozialform, die Medien, die erwartete Teilnehmerzahl und/oder die angestrebte Dauer eingegrenzt werden. Die so erschlossenen Methoden werden in Form einer tag cloud angezeigt, die im Zuge der Auswahl ihre Anordnung verändert: Die unter didaktischen Gesichtspunkten besten Treffer wandern in die Mitte, weniger passende an den Rand. Das Auswahlmenü unterstützt interdependente Planungsentscheidungen auch dadurch, dass es schrittweise genutzt werden kann. So können Unterrichtsmethoden sukzessive in Abhängigkeit von bereits getroffenen Entscheidungen über Lernziele, Gruppengröße usw. identifiziert werden. 
Abb. 2 Darbietung von Methoden in Form von Text und Video. (Quelle: Eigene Darstellung)

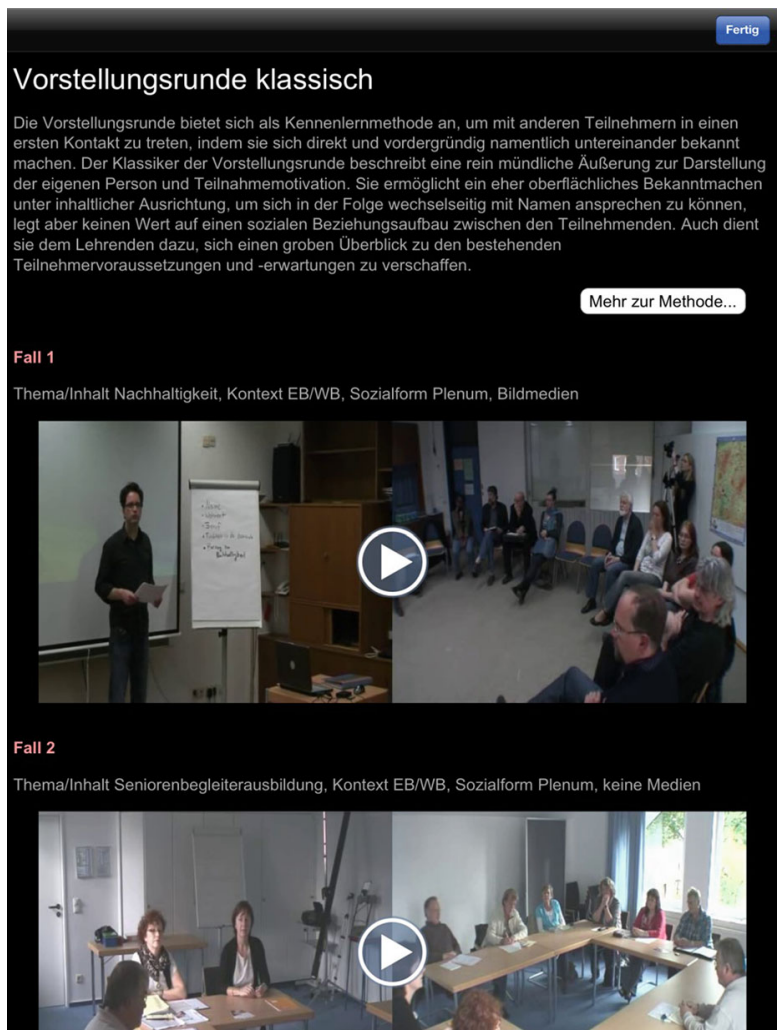

Eine zweite Funktionalität dient dazu, den extraneous cognitve load im Planungsprozess zu reduzieren, indem die Anschaulichkeit der verfügbaren Methoden erhöht wird und damit auch Einschätzungen ihrer möglichen Wirksamkeit in der erwarteten Lerngruppe unterstützt werden. Dazu bietet die App - wie die gängige Methodenliteratur auch - kurze Texte zu ausgewählten Methoden. Ergänzend werden authentische Videomaterialien angeboten, die anschaulich sind und damit Einschätzungen zu ihrer Umsetzbarkeit unter den je konkreten Rahmenbedingungen verbessern können. Die angebotenen Videofälle können zudem helfen, situative Bezüge herzustellen (vgl. Digel et al. 2012; Abb. 2).

Eine dritte Funktionalität soll den germane cognitive load reduzieren, indem kreative Suchbewegungen durch intuitive Bedienung und flexible Kombinationsmöglichkeiten unterstützt und damit sowohl top-down- als auch bottom-up-Prozesse bei der Unterrichtsplanung ermöglicht werden (vgl. Moreno und Valdez 2005). So kann ein Einstieg in die Erschließung geeigneter Methoden über einen direkten Zugriff auf einzelne Methoden gewählt werden. Über ein Antippen des Namens der Methode in der tag-cloud-Ansicht wird die gewählte Methode anhand eines Kurzabstracts vorgestellt und kann entweder über eines der zwei bis vier kurzen authentischen Unterrichtsvideos mit exemplarischen Gestaltungs- und Umsetzungsformen oder in Form eines einseitigen Methodentextes näher erschlossen werden. Der Einstieg kann aber auch - wie von vielen didaktischen Theorien empfohlen - zunächst über die 
Lernziele und dann über die weiteren verfügbaren didaktischen Auswahlkriterien erfolgen. Auf diese Weise können unterschiedliche Unterrichtsentwürfe in kurzer Zeit erprobt und in ihrer „Passung“ zur konkreten Lehr-Lern-Einheit gegeneinander abgewogen werden (Abb. 2).

Weitere, hier nicht näher beschriebene Funktionalitäten der App sehen vor, dass Planungsprozesse auch ausgehend von einer Übersicht zur Kursablaufplanung gestartet und darin dann einzelne Methoden als Bausteine importiert werden können. Zudem lassen sich einmal in den Verlaufsplan als Kursbausteine integrierte Methoden kommentieren und mit Notizen versehen. Bei gleichen (didaktischen) Auswahlkriterien kann die Reihenfolge der Methoden beliebig getauscht und damit der Gesamtablauf verändert werden. Zudem erlaubt es die App, die Planung schrittweise durchzuführen, jederzeit zu unterbrechen, Teilschritte zu speichern und zu einem späteren Zeitpunkt gegebenenfalls zu revidieren. Die mediale Gestaltung des Planungstools in Form einer App sichert zudem ihre ortsunabhängige Anwendbarkeit, da die präsentierten Inhalte auch offline genutzt werden können.

Diese und weitere technische Ausgestaltungen sollen Lehrkräfte bei der Informationsverarbeitung (Berücksichtigung von Interdependenzbeziehungen, Sicherung von Anschaulichkeit, Ermöglichung iterativer Entscheidungsprozesse) kognitiv entlasten. Die Erwartung besteht darin, dass die ,frei“ gewordenen kognitiven Kapazitäten dann für didaktisch reflektierte Entscheidungen genutzt werden können.

\section{Fragestellungen und Befunde einer quasi-experimentellen Feldstudie}

In einer quasi-experimentellen Feldstudie wurde untersucht, ob (1) die entwickelte App Lehrende bei der Kursplanung kognitiv entlasten und (2) dadurch die didaktische Qualität von Unterrichtsentwürfen verbessert werden kann. Die Studie wurde in das reguläre Angebot des Bachelorstudiengangs Erziehungswissenschaft der Universität Tübingen integriert und im Sommersemester 2014 durchgeführt. Thematisch ging es in dem Seminar um die mikrodidaktische Planung und Evaluation von Veranstaltungen der Erwachsenenbildung; dieses Seminar wurde bei gleichen Inhalten und gleicher Organisationsform insgesamt drei Mal angeboten.

Der Studie lag ein Experimental-Kontrollgruppen-Design zugrunde. Die Studierenden erhielten die Aufgabe, Kurseinstiege zu konzipieren, zu präsentieren und zu begründen. Als abhängige Variable wurden die Planungsfähigkeiten der Teilnehmenden erfasst. Die verfügbaren Planungshilfen wurden als unabhängige Variable wie folgt variiert: Gruppe 1 ,App“ $(N=16)$ erhielt das skizzierte KursplanungsTool, Gruppe 2 ,Print“ $(N=16)$ arbeitete mit den gleichen Darstellungen der Methoden zur Gestaltung von Anfangssituationen in Printform (also ohne Videos) (in Anlehnung an Müller et al. 2012), und Gruppe 3 „Kontrolle“ $(N=18)$ erhielt keine Planungshilfen.

Die Umsetzung erfolgte in einer 60-minütigen Partnerarbeit. Die Aufgabe bestand darin, einen maximal dreistündigen Kurseinstieg für eine zweitägige Einzelveranstaltung in Präsenzform zu entwerfen. Die Kleingruppen wurden aufgefordert, einen Entwurf für den Einstieg in einen Kurs zu erarbeiten, der sich dem Thema „Kommunikation und Interaktion für Führungskräfte“ widmete und bei dem die Gruppe 
der Teilnehmenden der Lehrkraft unbekannt war. Die Studierenden sollten während des Planungsprozesses „laut denken“ und wurden bei der gemeinsamen Planung videografiert. Direkt im Anschluss an die Erarbeitung einer Kursplanung wurden die Teilnehmenden standardisiert befragt. Der dabei eingesetzte Fragebogen orientierte sich an der Cognitive Load Theory und umfasste überwiegend geschlossene Fragen im Likert-Format ( 1 = trifft nicht zu bis $4=$ trifft $\mathrm{zu}$ ) sowie ergänzend offene Antwortmöglichkeiten. Der Fragebogen enthielt Itembatterien zur subjektiven Einschätzung der mentalen Belastung (z. B. „Ich habe mich bei der Aufgabe mental angestrengt“), der Schwierigkeit der Aufgabe (z. B. „Die Aufgabe war für mich schwer zu lösen“), der Verständlichkeit der Planungshilfen (z. B. „Die Methoden wurden verständlich dargeboten“) oder der Steuerungsmöglichkeiten des Planungsprozesses (z. B. „Ich konnte den Planungsprozess als Ganzes überblicken“). Für die Auswertungen wurden für jede Kategorie Mittelwerte berechnet.

Im Anschluss wurden die Planungsentwürfe von den Zweiergruppen in Form einer zehnminütigen Präsentation vorgestellt und abschließend in Einzelarbeit auf Basis von einheitlichen Reflexionsfragen begründet. Die Fragen lauteten: (1) Bitte begründen Sie, welche Ziele Sie mit der Gestaltung der Einstiegssituation erreichen möchten? (2) Warum haben Sie sich für die ausgewählten Methoden entschieden? (3) Warum nicht für andere, die auch hätten gewählt werden können? Die Präsentationen fanden einzeln gegenüber der Seminarleitung und somit unabhängig voneinander statt, sodass die Gruppen nicht voneinander lernen konnten.

Der Entwurf der Kursplanung sowie die erfragten Begründungen wurden inhaltsanalytisch codiert, wobei sich die Kategorien für die Begründungen aus den Reflexionsfragen ableiteten. Die Qualität der Kursentwürfe wurde anhand folgender Kriterien beurteilt: (1) Plausibilität des Aufbaus vor dem Hintergrund gesetzter Ziele; (2) Angemessenheit der Methoden im Blick auf die sachliche, psychologische und soziale Dimension zur Aktivierung der Teilnehmenden (vgl. Siebert 2006); (3) Vollständigkeit und innere Kohärenz der Kursplanungen in Bezug auf die berücksichtigten didaktischen Planungsfaktoren (Ziele, Inhalt, Methoden, Medien und Erfolgskontrolle im Blick auf die definierte Zielgruppe und die formulierten Rahmenbedingungen) (vgl. Schlutz 2006). Die Beurteilung erfolgte in Form eines vierstufigen Ratings (bspw. 1 = nicht vollständig bis 4 = vollständig), das von einer Projektmitarbeiterin sowie von einem Didaktikexperten unabhängig voneinander vorgenommen wurde. Die auf Basis von Krippendorffs Alpha berechnete Übereinstimmung weist dabei gute bis sehr gute Werte auf (z. B. $\alpha=0,78$ für die Plausibilität der Kursentwürfe; vgl. Wirtz und Caspar 2002).

Die Darstellung der Ergebnisse bezieht sich im Folgenden auf die Daten aus den quantitativen Befragungen sowie auf die inhaltsanalytische Auswertung der Kursentwürfe und deren Begründung.

\subsection{Stichprobe}

An der quasi-experimentellen Feldstudie haben insgesamt 50 angehende Lehrkräfte teilgenommen. Diese weisen ein Durchschnittsalter von 21 Jahren auf und sind zu $90 \%$ weiblich. Alle haben Erwachsenen- und Weiterbildung als Studienschwerpunkt im Bachelorstudiengang Erziehungswissenschaft an der Universität Tübingen 
gewählt, $70 \%$ studieren im Haupt- und $30 \%$ im Nebenfach, alle sind in Vollzeit in den Studiengang eingeschrieben. Die Teilnehmenden haben zu $96 \%$ keine Berufserfahrung als Lehrkraft in der Erwachsenen- und Weiterbildung, $4 \%$ sind nebenberuflich in geringem Umfang als Kursleitende mit (jungen) Erwachsenen tätig. Ihre didaktisch-methodischen Vorkenntnisse stufen die Studierenden durchschnittlich als wenig ausgeprägt ein $(M=1,7 ; S D=0,586)$. Mit der Lektüre von Methodenliteratur hingegen schätzen sie sich als eher vertraut ein $(M=3,1 ; S D=0,646)$. Gleichzeitig stellt diese für sie keine adäquate Planungshilfe dar $(\mathrm{M}=1,4 ; \mathrm{SD}=0,512)$, vielmehr fällt es ihnen nach Selbsteinschätzung tendenziell eher schwer, die ,theoretischen“ Inhalte in (künftiges) praktisches Handeln zu übersetzen $(\mathrm{M}=1,7 ; \mathrm{SD}=0,69)$.

\subsection{Zentrale Befunde}

Die Ergebnisse beziehen sich auf 50 Teilnehmende bzw. 25 Kursentwürfe, die in Zweiergruppen erstellt wurden. Die Befunde werden in Form von Häufigkeitsangaben und Mittelwertberechnungen je Gruppe „App“, „Print“ und „Kontrolle“ berichtet, zudem werden die Gruppen in Bezug auf ihre Einschätzungen der Kursplanung anhand einer einfaktoriellen Varianzanalyse verglichen.

\subsubsection{Befragungsdaten zur subjektiven Einschätzung der Kursplanung}

Im Blick auf die Einschätzung der Schwierigkeit der Aufgabe gab es keine signifikanten Differenzen zwischen den drei untersuchten Gruppen. Die gestellte Aufgabe in Form eines Entwurfs für den Einstieg in einen zweitägigen Kurs zum Thema „Interaktion und Kommunikation für Führungskräfte“ stuften ca. drei Viertel der 50 Teilnehmenden als komplex sowie inhaltlich anspruchsvoll ein. Dabei konnten $17 \%$ der Teilnehmenden in der Planung auf praktisches Wissen zur Gestaltung von Einstiegssituationen zurückgreifen; die dargebotenen Methoden waren $28 \%$ der Teilnehmenden überwiegend bekannt. Hingegen bestanden zwischen den drei Gruppen signifikante Unterschiede bezüglich der subjektiv empfundenen kognitiven Belastung $(\mathrm{F}(2,48)=28,37 ; p<0,01)$. Diese war bei der Gruppe „Print“ am höchsten und in der Gruppe „App“ am niedrigsten ausgeprägt $(\mathrm{M}=3,4 ; \mathrm{SD}=0,456$; versus Gruppe „App“ mit $\mathrm{M}=2,6 ; \mathrm{SD}=0,327$ und Gruppe „Kontrolle“ mit $\mathrm{M}=2,9$; $\mathrm{SD}=0,395)$. Vergleichbare signifikante Unterschiede ergaben sich auch bezüglich der Einschätzung der Klarheit der Planungshilfen $(\mathrm{F}(2,48)=24,71 ; p<0,01)$. So fanden die Mitglieder der Gruppe „App“ die digitale Fassung der Arbeitsvorlagen und Unterstützungshilfen deutlich klarer gestaltet $(\mathrm{M}=3,6 ; \mathrm{SD}=0,398)$ als die Gruppen „Print“ $(M=2,3 ; \mathrm{SD}=0,404)$ und „Kontrolle“ $(\mathrm{M}=2,1 ; \mathrm{SD}=0,576)$. Die Gruppen „Print“ und „Kontrolle“ hatten zudem in einem signifikant geringerem Ausmaß das Gefühl, die Einsatzmöglichkeiten der ihnen bekannten bzw. in Printform vorliegenden Methoden gut beurteilen zu können $(\mathrm{M}=2,4$; $\mathrm{SD}=0,513$ bzw. $\mathrm{M}=2,3$; SD = 0,634), während die Gruppe „App“ den Transfer auf Basis der als Text und Video vorliegenden Methoden optimistischer einschätzte $(M=3,4$; $\mathrm{SD}=0,422 ;(\mathrm{F}(2,48)=26,11 ; p<0,01))$. Weiter bestanden zwischen den Gruppen signifikante Unterschiede in Bezug auf die Begründung des erstellten Kursentwurfs $(\mathrm{F}(2,48)=22,56 ; p<0,01)$. So konnten die Mitglieder der Gruppen „Print“ sowie 


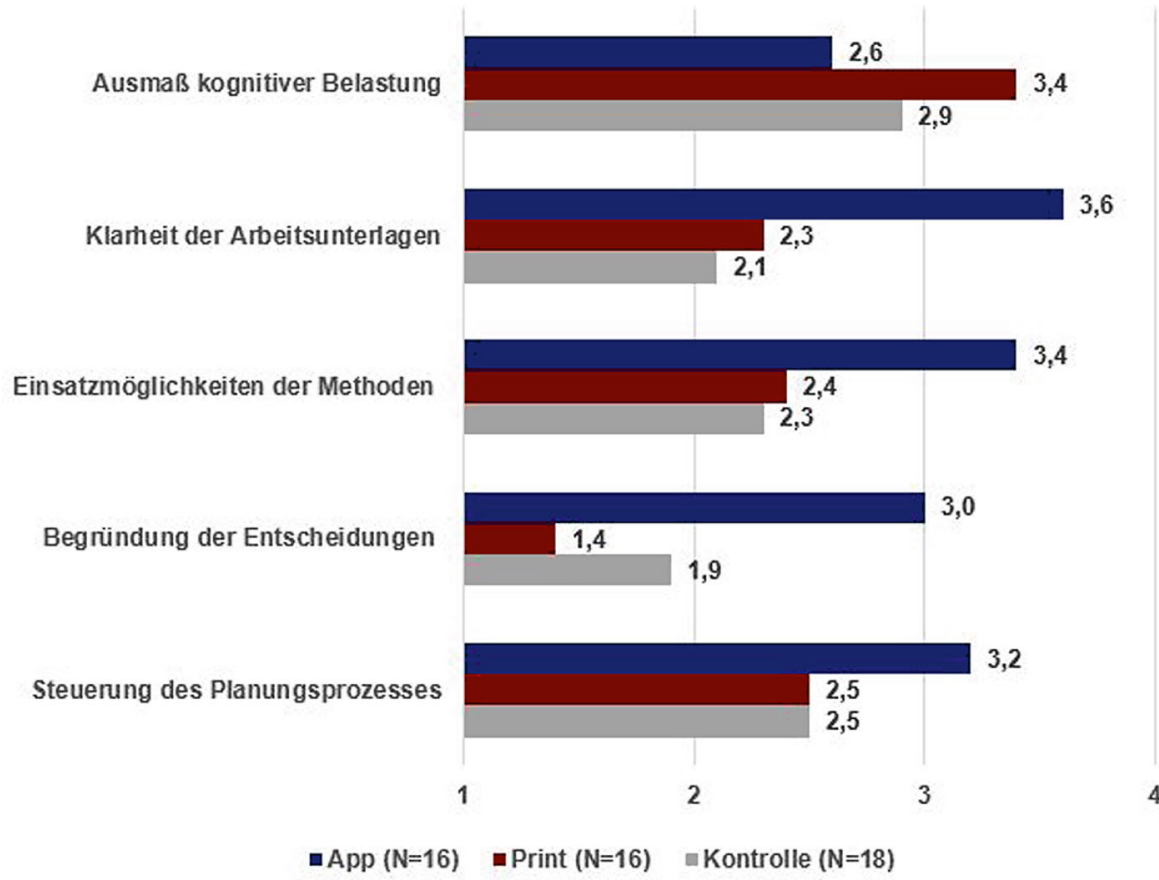

Abb. 3 Mittelwertvergleich der drei Gruppen zur subjektiven Einschätzung der Kursplanung. (Quelle: Eigene Berechnung)

„Kontrolle“ ihre Methodenauswahl kaum begründet treffen $(\mathrm{M}=1,4 ; \mathrm{SD}=0,423$ bzw. $\mathrm{M}=1,9 ; \mathrm{SD}=0,568$ ), während die Mitglieder der Gruppe „App“ eher zu einer Begründung ihrer Entscheidungen in der Lage waren $(M=3,0 ; S D=0,601)$. Auch in der Einschätzung der eigenen Steuerungsmöglichkeiten des Planungsprozesses traten signifikante Unterschiede auf $(\mathrm{F}(2,48)=20,21 ; p<0,01)$. Die Einschätzung fiel in der Gruppe „App“ am positivsten aus ( $\mathrm{M}=3,2$; $\mathrm{SD}=0,513$ versus Gruppe „Print“ und „Kontrolle“ jeweils mit Mittelwerten um M = 2,5) (Abb. 3).

Zusammenfasend betrachtet kann somit festgehalten werden, dass die Gruppe „App“ eine geringere kognitive Belastung zum Ausdruck brachte als die beiden anderen Gruppen; diese Entlastung scheint sich insbesondere auf die Verringerung des extraneous und intrinsic cognitive load zu beziehen. Die Gruppe „App“ konnte zudem im Vergleich zu den beiden anderen Gruppen didaktisch fundiertere Entscheidungen treffen und häufiger situative Bezüge herstellen.

\subsubsection{Reflexionsfragen zur Begründung des Kursentwurfs}

Die Begründung des Kursentwurfs vor dem Hintergrund der vorgegebenen Ziele stellte alle 50 Teilnehmenden unabhängig von ihrer Gruppenzugehörigkeit vor eine ungewohnte Herausforderung. Nur ca. 50\% der Antworten stellten eine didaktische Begründung der Kursgestaltung dar; diese bezog sich wiederum zu ca. einem Drittel auf eine Passung der Methode zu den thematisierten Inhalten und ihrer Vermittlung 
und Erarbeitung. Nur ca. ein Viertel der Studierenden begründete die methodischen Auswahlentscheidungen für den Kurseinstieg mit dem Verweis auf adressierte Lernziele. Dabei gehörten 10 dieser 12 Studierenden der Gruppe „App“ an.

Konkreter nach den Gründen für die Wahl der in den Kursentwurf einbezogenen Methoden befragt, wurden von den Studierenden unabhängig von ihrer Gruppenzugehörigkeit die Bekanntheit der Methode (30\%) und die subjektive Einstellung gegenüber der Methode (26\%) am häufigsten als Auswahlkriterien genannt. Darauf folgten Argumente zur Passung der Methode zu den Inhalten $(21 \%)$ sowie deren Eignung zur Gruppenbildung (17\%). Ferner nannten $16 \%$ der Teilnehmenden die überzeugende Darstellung einer Methode und ihrer Umsetzung als Grund für die Auswahl. Dabei entfielen alle dieser Kategorie zugeordneten Äußerungen auf die Mitglieder der Gruppe „App“, weiter brachten diese auch als einzige das Argument der situativen Eignung der Methode in $10 \%$ der Äußerungen als Begründung für die Auswahl ein.

Andere Methoden, die auch für die Kursplanung hätten gewählt werden können, wurden in erster Linie aufgrund von Unvertrautheit (17\%) und Unbeliebtheit (32\%) ausgeschlossen. Aus Sicht von $15 \%$ der Studierenden ermöglichten diese Methoden zudem das Erreichen einer sozialen Zusammenführung der Teilnehmenden nicht bzw. weniger gut. Ergänzend wurde die nicht vorhandene Passung der Methoden zueinander, zur Gruppengröße und zu den organisationalen Rahmenbedingungen genannt. Diese Ausschlusskriterien wiederum wurden zu $90 \%$ von Mitgliedern der Gruppe „App“ genannt.

Zusammenfassend betrachtet fällt auf, dass es allen Studierenden schwerfällt, ihre Kursentwürfe didaktisch zu begründen. Allein die Mitglieder der Gruppe „App“ nannten das Erreichen bestimmter Lernziele als Grund für ihre Unterrichtsentwürfe. Darüber hinaus hatte die Gruppe „App“ intensiver über die Eignung von Methoden nachgedacht und häufiger fallbezogene Auswahlentscheidungen getroffen.

\subsubsection{Beurteilung der Kursentwürfe}

Trotz der einheitlich gestellten Planungsaufgabe lagen 25 sehr unterschiedliche Kursentwürfe vor. Durch die Festlegung der Kurseinstiege auf die maximale Dauer eines Vormittags, ist der grundsätzliche Umfang von durchschnittlich zwei Stunden recht vergleichbar, während die Anzahl der enthaltenen Einheiten sowie die Zahl der darin einbezogenen Methoden zwischen zwei bis vier variierten.

Betrachtet man den Aufbau der Kursentwürfe, fällt auf, dass die Ziele zumeist als Grobziele und übergreifend für die gesamte Dauer des Einstiegs und damit mehrere Einheiten formuliert wurden. Wurden Ziele für einzelne Einheiten bzw. Schritte formuliert, waren diese zumeist gezielter auf die soziale Dimension der Gestaltung von Einstiegssituationen bezogen, ohne aber die unterschiedlichen Dimensionen von Einstiegssituationen zu hierarchisieren. Somit erschien der Aufbau vor dem Hintergrund der formulierten Ziele nur in einzelnen Fällen plausibel, überwiegend wurden die formulierten Ziele nicht aufeinander bzw. konkret auf die schrittweise Kursgestaltung bezogen. Insgesamt wurden daher nur sechs von 25 Kursplanungen als durchgehend plausibel eingestuft, 14 Verlaufsplanungen wurden nur in einzelnen Teilschritten aufeinander bezogen, ohne dass allerdings ein durch die Ziele aufge- 
spannter und die Einstiegssituation übergreifender roter Faden erkennbar gewesen wäre. In den übrigen fünf Kursentwürfen fehlte ein solcher Spannungsbogen ganz. Die nicht bzw. wenig zielbezogen gestalteten Entwürfe stammten durchweg aus der Gruppe „Kontrolle“, vier der fünf zielbezogenen Ablaufpläne ließen sich der Gruppe „App“ zuordnen.

Dabei waren in allen Planungen eine Ablaufvorstellung sowie eine Methode zum gegenseitigen Kennenlernen der Teilnehmenden enthalten. An weiteren Methoden wurden Arbeitsformen zur kognitiven Aktivierung sowie dem persönlichen Ankommen der Teilnehmenden eingesetzt. Während somit alle Planungen die soziale Dimension der Gestaltung von Einstiegssituationen berücksichtigten, wurden die psychologische und die sachliche Dimension nur in Teilen einbezogen: Dies geschah jeweils nur bei einem Drittel der vorliegenden 25 Planungsentwürfe. Etwa ein Viertel der Planungsentwürfe thematisierte alle drei in Einstiegssituationen relevanten Gestaltungsdimensionen gleichermaßen (vgl. Siebert 2006). Diese entstammten mehrheitlich der Gruppe, deren Kursentwürfe mit Hilfe der App realisiert wurden. Zwei der ganzheitlichen Planungsentwürfe wurden in der Kontrollgruppe erstellt.

Analysiert man die einzelnen Phasen bzw. Einheiten hinsichtlich der darin berücksichtigten didaktischen Planungsfaktoren, können sechs Kursentwürfe unter Einschränkung der Feindifferenzierung der Ziele als eher vollständig bis vollständig eingestuft werden. Diese berücksichtigten die Methoden als Wegbereiter zur Erarbeitung der thematisierten Inhalte und enthielten Erfolgskontrollen, die in die einzelnen Phasen integriert wurden oder zumindest am Schluss des Kurseinstieges standen. Weiter erschienen die Bezüge bei zwei Drittel dieser Entwürfe auch kohärent. Während die kohärent geplanten Entwürfe bis auf einen Entwurf (aus der Gruppe „Kontrolle“) alle von Mitgliedern der Gruppe „App“ erstellt wurden, entstammten die restlichen zwei (eher) vollständigen Entwürfe der Gruppe „Kontrolle“. Die weniger vollständigen Ablaufplanungen enthielten nicht in jeder Phase klare Bezüge zwischen den Inhalten und Methoden. Als unvollständig wurden die Entwürfe eingestuft, bei denen zusätzlich noch Erfolgskontrollen für jede Phase fehlten und somit in keiner Phase die vier didaktischen Planungsfaktoren im Zusammenhang bedacht wurden. Dies traf auf vier der Kursentwürfe zu, die zur Hälfte den Gruppen „Print“ und „Kontrolle“ entstammten. Dabei wurden alle (eher) unvollständigen Kursentwürfe auch als nicht kohärent eingestuft.

Zusammenfassend betrachtet zeigt sich, dass die Mitglieder der Gruppe „App“ am häufigsten vollständige und kohärente Planungsentwürfe entwickelten, während dies der Gruppe, die mit der traditionellen Methodenliteratur arbeitete, seltener gelang. Damit scheint die App eine Unterstützung anzubieten, um bei der Planung in Zusammenhängen zu denken und verschiedene didaktische Planungsfaktoren integriert zu berücksichtigen. Dadurch wurden qualitativ hochwertigere Kursentwürfe erstellt, die in den Zielen, in den Inhalten, in den Methoden und der Erfolgskontrolle klarer aufeinander bezogen waren und somit in sich stimmig erschienen (vgl. Schlutz 2006). 


\section{Zusammenführung und Fazit}

Mit den Ergebnissen dieser ersten evaluativen Feldstudie zum Einsatz digitaler Medien bei der Planung von Lehr- Lern-Einheiten lassen sich die beiden Untersuchungsfragestellungen wie folgt beantworten: Die Nutzung der App führte bei angehenden Lehrenden zu einer Reduktion der subjektiv empfundenen kognitiven Belastung bei der Planung. Weiterhin begünstigte die in der App realisierte vernetzte Darstellung der Planungsmaterialien eine Entwicklung didaktisch reflektierter Kursentwürfe. Ein Planungsvorgehen, das von den begründeten Zielsetzungen eines Kurses ausgeht, konnte die App hingegen bei der hier berücksichtigten Adressatengruppe nicht durchgängig fördern. Dabei ist allerdings in Rechnung zu stellen, dass die Studierenden kaum über didaktisches Vorwissen sowie Erfahrungen in der Kursplanung verfügten. Dies zeigte sich auch in den Begründungen der Kursentwürfe, die überwiegend auf subjektiven Wertungen beruhten. Dennoch war die Qualität der Kursentwürfe, die auf Basis der App erstellt wurden, durchschnittlich höher als die der beiden Vergleichsgruppen. Im Ergebnis lagen dann, wenn die App verwendet wurde, eher vollständige und kohärente Planungen vor, die sowohl verschiedene didaktische Planungsfaktoren und deren Zusammenwirken berücksichtigten als auch neben der sozialen die psychologische und sachliche Dimension in die Gestaltung des Lehr-Lern-Geschehens beim Kurseinstieg einbezogen.

Allerdings unterliegen die vorliegenden Befunde auch einigen Einschränkungen. Zwar deutet sich an, dass die Nutzung der App angehende Lehrkräfte bei ihren Planungsaufgaben kognitiv entlastet und damit eine didaktisch reflektierte Kursplanung unterstützt. Auf welche Elemente und Funktionalitäten der App dies im Detail zurückgeführt werden kann, lässt sich anhand der vorliegenden Daten allerdings noch nicht sagen, so etwa weil die subjektiven Einschätzungen der kognitiven Belastung sich auf die gesamte Planungseinheit und nicht auf einzelne Phasen bzw. auf einzelne Funktionalitäten der App bezogen. Zudem war es aufgrund der Umsetzungsbedingungen im universitären Lehrbetrieb nicht möglich, eine BaselineErhebung der bereits vorhandenen Planungskompetenzen zu realisieren. Auch fehlt es noch an Follow-up-Daten, um einschätzen zu können, ob mit der hier realisierten Kurzzeit-Intervention didaktische Reflexivität nachhaltig gefördert werden konnte oder diese auf das durchgeführte Seminar beschränkt blieb. Schließlich steht die Analyse der Prozessdaten noch aus, um genauer beurteilen zu können, welchen Einfluss die Qualität der Zusammenarbeit in den Kleingruppen auf die Qualität der Unterrichtsentwürfe hatte. In anschließenden Forschungsarbeiten verdient auch die Frage Beachtung, wie viel professionelle Expertise - etwa im Vergleich von Novizen und Experten - bei den Lehrkräften bereits vorhanden sein muss, um die erweiterten Planungsmöglichkeiten optimal zu nutzen.

Gleichwohl erscheint es uns auf Basis der hier präsentierten, ersten Ergebnisse lohnenswert, an der Weiterentwicklung der Kursplanungs-App zu arbeiten. Die eingangs geschilderten empirischen Befunde zu Bedarfen und Interessen des Lehrpersonals an wissenschaftlich fundierten, ökonomisch, örtlich und zeitlich flexibel einsetzbaren Planungshilfen bestärken uns in solchen Überlegungen. Dazu gehört u. a. ihre Anreicherung um Methoden für jene Phasen von Lehr-Lern-Prozessen, die an den Einstieg anschließen, oder auch für spezifische Inhalts- und Fachbereiche 
(wie z. B. den (Zweit-)Spracherwerb oder das Training formaler Schlüsselqualifikationen). Darüber hinaus werden wir nach Wegen suchen, die Verbreitung der App in der Weiterbildungspraxis zu fördern, möglichst in Kombination mit dem DIE-Portal wb-web. Perspektivisch soll das entwickelte digitale Tool aber nicht nur einen Beitrag zur Unterstützung der Praxis leisten, indem es z. B. in Aus- und Weiterbildungskurse für Lehrkräfte integriert wird, sondern auch in Interventions- und Implementationsstudien zur Erforschung der Möglichkeit, die Professionalität des Lehrhandelns zu fördern, eingesetzt werden.

Open Access Dieser Artikel wird unter der Creative Commons Namensnennung 4.0 International Lizenz (http://creativecommons.org/licenses/by/4.0/deed.de) veröffentlicht, welche die Nutzung, Vervielfältigung, Bearbeitung, Verbreitung und Wiedergabe in jeglichem Medium und Format erlaubt, sofern Sie den/die ursprünglichen Autor(en) und die Quelle ordnungsgemäß nennen, einen Link zur Creative Commons Lizenz beifügen und angeben, ob Änderungen vorgenommen wurden.

\section{Literatur}

Breloer, G., Dauber, H., \& Tietgens, H. (1980). Selbststeuerung und Teilnehmerorientierung in der Erwachsenenbildung. Braunschweig: Westermann.

Bromme, R. (1997). Kompetenzen, Funktionen und unterrichtliches Handeln des Lehrers. In F. E. Weinert (Hrsg.), Psychologie des Unterrichts und der Schule. Enzyklopädie der Psychologie, Bereich D: Praxisgebiete, Serie I: Pädagogische Psychologie (Bd. 3, S. 177-212). Göttingen: Hogrefe.

Deutsches Institut für Erwachsenenbildung (DIE), Bundesinstitut für Berufsbildung (BIBB), \& Universität Duisburg-Essen (UDE) (2016). Das Personal in der Weiterbildung. Arbeits- und Beschäftigungsbedingungen, Qualifikationen, Einstellungen zu Arbeit und Beruf. Bielefeld: Bertelsmann.

Digel, S., Goeze, A., \& Schrader, J. (2012). Aus Videofällen lernen - Einführung in die Praxis für Lehrkräfte, Trainer und Berater. Bielefeld: Bertelsmann.

Gerjets, P., Scheiter, K., \& Cierniak, G. (2009). The scientific value of cognitive load theory: a research agenda based on the structuralist view of theories. Educational Psychology Review, 21(1), 43-54.

Gruber, H. (2004). Kompetenzen von Lehrerinnen und Lehrern - Ein Blick aus der Expertiseforschung (Forschungsbericht Nr. 13). Regensburg: Universität Regensburg.

Haberzeth, E. (2010). Thematisierungsstrategien im Vermittlungsprozess. Empirische Analysen zum Umgang mit Wissen im Planungsprozess von Weiterbildungsangeboten. Baltmannsweiler: Schneider Hohengehren.

Heimann, P. (1962). Didaktik als Theorie und Lehre. Die Deutsche Schule, 54, 407-472.

Heimann, P., Otto, G., \& Schulz, W. (1965). Unterricht - Analyse und Planung. Hannover: Schroedel.

Hof, C. (2001). Konzepte des Wissens: eine empirische Studie zu den wissenstheoretischen Grundlagen des Unterrichts. Bielefeld: Bertelsmann.

Hoffmann, N. (2011). Ratgeber-Didaktik in Didaktik-Ratgebern. Portrait eines Selbstlernmediums aus erwachsenenpädagogischer Perspektive. Der pädagogische Blick, 19(1), 4-12.

Kade, J. (1989). Kursleiter und die Bildung Erwachsener: Fallstudien zur biographischen Bedeutung der Erwachsenenbildung. Bad Heilbrunn: Klinkhardt.

Kade, S. (1990). Handlungshermeneutik: Qualifizierung durch Fallarbeit. Bad Heilbrunn: Klinkhardt.

Kirschner, P. A. (2002). Cognitive load theory: implications of cognitive load theory on the design of learning. Learning and Instruction, 12(1), 1-10.

Lave, J., \& Wenger, E. (1991). Situated learning: legitimate peripheral participation. Cambridge: University of Cambridge.

Martin, A., \& Langemeyer, I. (2014). Demographie, sozioökonomischer Status und Stand der Professionalisierung - das Personal der Weiterbildung im Vergleich. In Deutsches Institut für Erwachsenenbildung (Hrsg.), Trends der Weiterbildung: DIE-Trendanalyse 2014 (S. 43-88). Bielefeld: Bertelsmann.

Mayer, R.E., \& Anderson, R.B. (1992). The instructive animation: Helping students build connections between words and pictures in multimedia learning. Journal of Educational Psychology, 84(4), $444-452$. 
Mayer, R.E., \& Moreno, R. (2010). Techniques that reduce extraneous cognitive load and manage intrinsic cognitive load during multimedial learning. In J.L. Plass, R. Moreno \& R. Brünken (Hrsg.), Cognitve load theory (S. 131-152). Cambridge: University Press.

Michel, L.P. (2008). Digitale Schule - wie Lehrer Angebote im Internet nutzen. Tübingen: MMB-Institut für Medien- und Kompetenzforschung. http://www.dlr.de/pt/Portaldata/45/Resources/a_dokumente/ bildungsforschung/MMB_Veroeffentlichung_Lehrer_Online_20080505_final.pdf. Zugegriffen: 28. Nov 2016.

Moreno, R., \& Valdez, A. (2005). Cognitive load and learning effects of having students organize pictures and words in multimedial environments: the role of student interactivity and feedback. Educational Technology Research and Development, 53, 35-45.

Müller, M., Alsheimer, M., Iberer, U., \& Papenkort, U. (2012). Einführung in die Seminarplanung. Eine Spielanleitung mit Checklisten und Beispielen. Bielefeld: Bertelsmann.

Oser, F., \& Baeriswyl, F. (2001). Choreographies of teaching: bridging instruction to learning. In V. Richardson (Hrsg.), Handbook of research on teaching (4. Aufl., S. 1031-1065). Washington D.C.: AERA.

Sandfuchs, U. (1982). Unterrichtsvorbereitung - Bedeutung und Probleme, Hinweise und Ratschläge. In J. Baurmann, W. Menzel \& U. Sandfuchs (Hrsg.), Unterricht planen und vorbereiten. Praxis Deutsch Sonderheft 1982 (S. 4-9). Seelze: Friedrich.

Sandfuchs, U. (2009). Grundfragen der Unterrichtsplanung. In K.-H. Arnold, U. Sandfuchs \& J. Wiechmann (Hrsg.), Handbuch Unterricht (2. Aufl., S. 512-519). Bad Heilbrunn: Klinkhardt.

Schlutz, E. (2006). Bildungsdienstleistungen und Angebotsentwicklung. Münster: Waxmann.

Schnotz, W., \& Kürschner, C. (2007). A reconsideration of cognitive load theory. Educational Psychology Review, 19(4), 469-508.

Schöb, S., Sahlender, M., Brandt, P., Fischer, M., \& Wintermann, O. (2015). Information und Vernetzung Bedarfe und Erwartungen von Lehrkräften an online-gestützte Fortbildungsangebote. www.die-bonn. de/doks/2015-erwachsenenbildner-01.pdf. Zugegriffen: 28. Nov 2016.

Schrader, J. (2010). Fortbildung von Lehrenden der Erwachsenenbildung: Notwendig? Sinnvoll? Möglich? In J. Schrader, R. Hohmann \& S. Hartz (Hrsg.), Mediengestützte Fallarbeit. Konzepte, Erfahrungen und Befunde zur Kompetenzentwicklung von Erwachsenenbildnern (S. 25-68). Bielefeld: Bertelsmann.

Siebert, H. (2006). Didaktisches Handeln in der Erwachsenenbildung. Didaktik aus konstruktivistischer Sicht (5. Aufl.). Augsburg: ZIEL.

Stanik, T. (2016). Mikrodidaktische Planungen von Lehrenden in der Erwachsenenbildung - theoretische und empirische Annäherungen an ein Forschungsdesiderat. Zeitschrift für Weiterbildungsforschung. doi:10.1007/s40955-016-0076-7.

Sweller, J. (1994). Cognitive load theory, learning difficulty, and instructional design. Learning and Instruction, 4(4), 295-312.

Sweller, J., van Merrienboer, J., \& Paas, F. (1998). Cognitive architecture and instructional design. Educational Psychology Review, 10(3), 251-296.

Wiater, W. (1997). Unterrichten und lernen in der Schule. Eine Einführung in die Didaktik (2. Aufl.). Donauwörth: Auer.

Wiater, W. (2009). Didaktische Theoriemodelle und Unterrichtsplanung. In K.-H. Arnold, U. Sandfuchs \& J. Wiechmann (Hrsg.), Handbuch Unterricht (2. Aufl., S. 505-512). Bad Heilbrunn: Klinkhardt.

Wirtz, M., \& Caspar, F. (2002). Beurteilerübereinstimmung und Beurteilerreliabilität: Methoden zur Bestimmung und Verbesserung der Zuverlässigkeit von Einschätzungen mittels Kategoriensystemen und Ratingskalen. Göttingen: Hogrefe.

WSF - Wirtschafts- und Sozialforschung. (2005). Erhebung zur beruflichen und sozialen Lage von Lehrenden in Weiterbildungseinrichtungen: Schlussbericht. Kerpen: BMBF. 